Examining Three-way Binding as a Constraint on Statistical Learning

\author{
Hyungwook Yim \\ School of Psychological Sciences, The University of Melbourne, Australia \\ Simon J. Dennis \\ School of Psychological Sciences, The University of Melbourne, Australia \\ Vladimir M. Sloutsky \\ Department of Psychology, The Ohio State University, USA
}

word count: 6255 words

\begin{abstract}
In press: Journal of Experimental Psychology: Learning, Memory, and Cognition (C) 2019, American Psychological Association. This paper is not the copy of record and may not exactly replicate the final, authoritative version of the article. Please do not copy or cite without authors' permission. The final article will be available, upon publication, via its DOI: $10.1037 / x \operatorname{lm} 0000807$
\end{abstract}

\begin{abstract}
Author Note
This research was supported under Australian Research Council's (ARC) Discovery Projects funding scheme to SJD (DP150100272) and by the National Institutes of Health Grants R01HD078545 and P01HD080679 to VMS. Part of the study was presented at the 34th Annual Conference of the Cognitive Science Society. Data from this paper can be accessed through https://osf.io/48ymf. Correspondence concerning this article should be addressed to Hyungwook Yim, Melbourne School of Psychological Sciences, The University of Melbourne, Parkville, VIC 3010 Australia. E-mail: hyungwook.yim@unimelb.edu.au
\end{abstract}




\begin{abstract}
Models of statistical learning do not place constraints on the complexity of the memory structure that is formed during statistical learning, while empirical studies using the statistical learning task have only examined the formation of simple memory structures (e.g., two-way binding). On the other hand, the memory literature, using explicit memory tasks, has shown that people are able to form memory structures of different complexities and that more complex memory structures (e.g., three-way binding) are usually more difficult to form. We examined whether complex memory structures such as three-way bindings can be implicitly formed through statistical learning by utilizing manipulations that have been used in the paired-associate learning paradigm (e.g., AB/ABr condition). Through three experiments, we show that while simple two-way binding structures can be formed implicitly, three-way bindings can only be formed with explicit instructions. The results indicate that explicit attention may be a necessary component in forming three-way memory structures and suggest that existing models should place constraints on the representational structures that can be formed.
\end{abstract}

$172 / 250$ words

Keywords: three-way binding, statistical learning, constraint 


\section{Examining Three-way Binding as a Constraint for Statistical Learning}

Statistical learning has been identified as a powerful mechanism for implicitly extracting regularities in the environment across different domains such as segmenting words from a continuous auditory stream (e.g., Saffran, Aslin, \& Newport, 1996), learning artificial grammars (e.g., Reber, 1967), and extracting temporal or spatial patterns from visual input (e.g., Fiser \& Aslin, 2002; Kirkham, Slemmer, \& Johnson, 2002). Statistical learning exhibits an early onset in humans (Saffran, \& Kirkham, 2018) and has been proposed as a general learning mechanism underlying multiple aspects of human cognition such as perceptual/motor learning, categorization, memory, and language acquisition (Armstrong, Frost, \& Christiansen, 2016).

Major theories and models of statistical and implicit learning propose some variant of a chunking mechanism as a way of explaining the extraction process. For example, PARSER (Perruchet \& Vinter, 1998) assumes that different kinds of chunks are automatically parsed from the input and increases the weights for the chunks that are encountered more often. TRACX (French, Addyman, \& Mareschal, 2011; Mareschal \& French, 2017) gates the input pattern by examining the degree to which the adjacent input stimuli were experienced together, and simple recurrent networks (e.g., Elman, 1990) incorporate a prediction mechanism to find the boundaries between chunks. Although the detail process of chunking differs across models, most of the models assume that a representation of each chunk is formed. As a consequence, there are no theoretical constraints in these models on the complexity of representation that is formed since the chunking mechanism is flexibly defined -- once the elements are seen together frequently enough they form a unit (or chunk). 
On the other hand, memory research suggests that people are able to form different kinds of complex memory structures. In a laboratory setting, memory structures of different complexities have been often studied using the paired-associate learning task. In the task, participants study item pairs in multiple contexts (usually as multiple lists) and are later tested on how the items were paired in each context. Depending on the pairings of items in different contexts, a different level of representational complexity is required to later retrieve the events correctly. For example, in an $\mathrm{AB} / \mathrm{CD}$ condition, participants are shown item $\mathrm{A}$ and $\mathrm{B}$ paired in the first list and, C and D paired in the second list during the study phase -- the items in context1 (i.e., A and B) and their pairing (i.e., AB) are different from those in context2 (i.e., C and $\mathrm{D}$ paired as $\mathrm{CD}$ ). If a participant is asked which item was paired with $\mathrm{A}$ in the first list, at the minimum and assuming these are novel items, one would only have to form a representation structure that binds the two items (i.e., A and B) to answer correctly. However, in a structurally more challenging $\mathrm{AB} / \mathrm{ABr}$ condition (Porter \& Duncan, 1953; Aue, Criss, \& Novak, 2017; Wahlheim \& Jacoby, 2013), the items in context1 are identical to the ones in context2, but they are paired differently (e.g., context1 consists of the pairs A-B, and C-D, whereas context 2 consists of pairs $\mathrm{A}-\mathrm{D}$ and $\mathrm{C}-\mathrm{B})$. In the $\mathrm{AB} / \mathrm{ABr}$ condition, a correct retrieval requires one, at the minimum, to form three-way binding structures (Humphreys, Bain, \& Pike, 1989; Osth \& Dennis, 2015; Plate, 1995), which consist of a context and two items as a configural code (e.g., context1-A-B). When a participant is asked which item was paired with A in context1, a correct retrieval requires a compound cue consisting of the context and the cued item (e.g., context1-A) to retrieve the correct response (e.g., B). If only two-way bindings have been formed, at test, context1 would elicit item B and D since both have been 
encountered in context1, and item A would elicit item B and D as well since A has been paired with $\mathrm{B}$ in context1 and $\mathrm{D}$ in context2, which creates response ambiguity. Therefore, while multiple two-way bindings only provide information about the dyadic pair associations, three-way bindings additionally provide information about the three elements being coherently associated (see Yim, Osth, Sloutsky, \& Dennis, 2018 for an illustration).

Correctly retrieving information from an $\mathrm{AB} / \mathrm{ABr}$ condition could be thought of as an exclusive or (XOR) problem (Wiles \& Humphreys, 1993). In the XOR operation, which is expressed using the symbol $\stackrel{\vee}{ }$, when zero is combined with zero the answer is one (i.e., $0 \unrhd 0=$ 1), and zero combined with one is zero (i.e., $0^{\unrhd} 1=0$ ). When one is combined with zero the

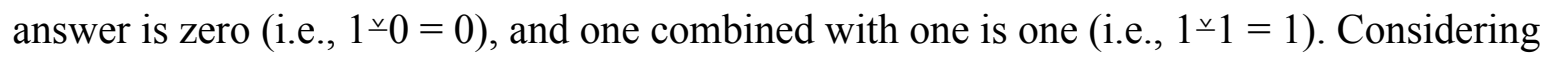
the first two terms as the cues at test (i.e., first term as the context cue, and second term as the item cue), and considering the answer of the XOR operation as the to-be-retrieved target, the process of retrieving an answer from the $\mathrm{AB} / \mathrm{ABr}$ condition becomes identical to the $\mathrm{XOR}$ problem. The solution of the XOR is well known to be impossible within a two-dimensional plane that has independent inputs using linear decision bounds (e.g., Minsky \& Papert, 1969), but can be solved by increasing the dimension of the inputs such as using multiplicative (configural) coding of the inputs (Sloman \& Rumelhart, 1992) or nonlinear decision bounds. Similarly, the $\mathrm{AB} / \mathrm{ABr}$ condition can not be solved with a two-way binding structure, in fact not even multiple two-way binding structures suffice (e.g., Humphreys, Bain, \& Pike, 1989). It requires a higher dimensional representation such as three-way binding structure.

Complex binding structures have also been discussed in terms of processing capacity and are required to build higher-level knowledge representations such as relational structures (e.g., 
Doumas, Hummel, \& Sandhofer, 2008; Halford, Wilson, \& Phillips, 1998). However, complex binding structures have only been studied through explicit tasks such as in reasoning or memory tasks. On the other hand, previous studies using statistical/implicit learning tasks have mainly provided evidence for forming two-way bindings (e.g., Gómez, 2002; Nissen \& Bullemer, 1987; Reber, 1967; Saffran, Aslin, \& Newport, 1996) since most statistical learning tasks use unique elements (e.g., A-B-C vs. E-F-G) instead of overlapping elements (e.g., A-B-C vs. A-B-D).

In Cohen, Ivry, and Keele (1990), an AB/ABr-like structure was used in a serial reaction time task. In the task, participants had to respond to a fixed sequence of 132312 (see Figure 1A), where each number corresponds to the position on the screen (e.g., 1=left, $2=$ middle, $3=$ right). In this sequence, all positions occur twice, and the sequence could not be predicted by an immediately preceding position (i.e., 1-back prediction). One way to predict the next position is to form compound cues (i.e., two preceding positions) to predict the next position (e.g., 13->2, 32->3, 23->1, etc.), which resembles the three-way binding solution for the $\mathrm{AB} / \mathrm{ABr}$ condition. Since adult participants showed evidence for learning in the task, the results could be interpreted to suggest that three-way binding structures could be learned without explicit attentional control. However, a careful examination of their task reveals that the sequence is not a pure $\mathrm{AB} / \mathrm{ABr}$ structure but rather a mixture of $\mathrm{AB} / \mathrm{ABr}$ and non- $\mathrm{AB} / \mathrm{ABr}$ structures. For example, a sequence of 1-3-2 requires a three-way binding structure, since the 1-back item (i.e., 3) predicts both items 1 and 2, while the 2-back item (i.e., 1) predicts both items 1 and 2 (see Figure 1B and 1C for all possible 1-back and 2-back predictions that could be learned through the sequence of their task and an illustration). Therefore, a compound cue 
(i.e., 1-3) that predicts the 3rd item (i.e., 2), which is a three-way binding structure, is required for a correct prediction. On the other hand, a sequence of 2-3-1 does not require a three-way binding structure. In the sequence, the 1-back item (i.e., 3) predicts 2 and 3, while the 2-back item (i.e., 2) predicts 1 and 3 (see Figure 1D for an illustration). By taking the intersection of the two predictions (i.e., 3) a correct prediction can be made. This structure resembles the $\mathrm{AB} / \mathrm{AC}$ condition of the paired-associate learning paradigm, where two two-way bindings and their intersection can generate a correct answer. Consequently, the task provides a mixture of $\mathrm{AB} / \mathrm{ABr}$ and $\mathrm{AB} / \mathrm{AC}$ structures, where among six possible triplet sequences, two require a three-way binding (i.e., 1-3-2 and 3-1-2), whereas four do not (i.e., 3-2-3, 1-2-1, 2-3-1, and 2-1-3). Therefore, given that there are ways to perform above chance without forming three-way bindings, the study by Cohen et al. (1990) does not unambiguously implicate three-way bindings.

Developmental evidence also questions whether complex three-way bindings can be formed implicitly. In Yim, Dennis, and Sloutsky (2013), 4-year-olds, 7-year-olds, and adults were tested using a paired-associate learning task with different structural conditions (i.e., $\mathrm{AB} / \mathrm{CD}, \mathrm{AB} / \mathrm{AC}, \mathrm{AB} / \mathrm{ABr})$. Using a multinomial processing tree model to infer representations from patterns of responses, they demonstrated that the ability to form three-way bindings undergoes substantial development between the age of seven and adulthood. Such protracted development suggested that the ability may hinge on attentional control during encoding, which also undergoes protracted development (Drummey \& Newcombe, 2002; Ghetti \& Bunge, 2012). Consequently, it was hypothesized that a key determinant of forming three-way binding structures may be explicit attentional control. If explicit attentional control is a core 
mechanism for forming three-way bindings, it is unlikely that three-way bindings can be formed implicitly.

Therefore, in the current study, we examined whether a complex binding structure such as a three-way structure can be formed implicitly through a statistical learning task. Finding evidence for forming three-way bindings would extend the kinds of representational structure that are known to be formed implicitly, while evidence against would provide a new constraint on models of statistical/implicit learning. To examine these issues, we applied the $\mathrm{AB} / \mathrm{ABr}$ structure to a statistical learning task (e.g., Saffran et al., 1996). In such tasks, stimuli have a certain structure (e.g., the presence of one stimulus is conditional upon the presence of previous stimuli), and participants are typically able to learn the structure without being aware that there is a structure. In Experiment 1, three-way binding structures were examined with two other two-way binding structures that have shown evidence of learning in previous studies (e.g., Gebhart, Newport, \& Aslin, 2009; Turk-Browne, Simon, \& Sederberg, 2012). In Experiment 2, we examine three-way bindings with a longer learning phase than Experiment 1, and in Experiment 3 we examined whether three-way bindings are learnable in the current task when explicit instructions are given to the participants.

\section{Experiment 1}

In order to examine the formation of three-way bindings in an implicit/statistical learning task, we utilized the $\mathrm{AB} / \mathrm{ABr}$ structure which has been used in the paired-associate learning task (see the Three-way column in Table 1). Each element of the triplet was represented as a cartoon character (see first row of Figure 2A). In the task, each image was presented one at a time, and participants were asked to make judgements about the gender of the character, which 
served as a cover task (see first row of Figure 2B). Unbeknownst to the participants, the presentation order of the images had a particular structure, and learning this structure was the underlying goal of the task.

In addition to the three-way condition using the $\mathrm{AB} / \mathrm{ABr}$ structure, there were three additional within-subjects conditions (see Figure 2, and also the method section). Each condition had a training phase and a test phase, with the transition between the phases being unannounced to the participants. The only difference between the training and test phase was in the underlying stimulus structure. The test phase consisted of a mixture of triplets in the training phase (i.e., congruent triplets) and triplets that do not follow the structure of the training phase (i.e., incongruent triplets). If the participants were able to learn the structure and extract the triplets in the training phase, performance for the congruent triplets should be better than for the incongruent trials. Using our procedure, we expect to see this difference manifested as response time to the third element, with the preceding two elements of the triplet serving as cues predicting the third element.

The use of an implicit-learning and implicit-testing design in the current study has a major advantage since it is always possible that what has been learned could be sensitive to the learning context, and changing the test context could hinder capturing the evidence of learning as previous literature has shown (Franco, Eberlen, Destrebecqz, Cleeremans, \& Bertels, 2015; Gómez, 1997; Siegelman, Bogaerts, Christiansen, \& Frost, 2017).

\section{Method}

Participants. One hundred and twenty-five undergraduates at The Ohio State University participated for course credit. Seven additional participants were excluded due to not following 
the instructions (e.g., pressing only one button, pressing more than two buttons throughout the experiment, or self-reporting that they misunderstood the task; $N=6)$, or device error $(N=1)$. The study was approved by The Ohio State University's Institutional Review Board.

Materials and Design. The stimuli were thirty-six pictures of cartoon characters, with half being male, and the other half female. There were four within-subject conditions (i.e., three-way, non-adjacent two-way, adjacent and non-adjacent two-way, and baseline). In the three-way condition, the structure of the training triplets resembled the $\mathrm{AB} / \mathrm{ABr}$ condition in the paired-associate learning task (see Table 1). The last items in the sequences (i.e., $B$ or $D$ ) could not be predicted by either the second items (i.e., $A$ or $C$ ) or the first items (i.e., $X_{1}$ or $X_{2}$ ), and required both items to be formed into a compound cue (e.g., $X_{1} A->B$ ) to predict whether the third item was B or D. In the non-adjacent two-way binding condition, training triplets were structured so that only the first items (i.e., $X_{3}$ and $X_{4}$ ) predict the third items (i.e., $F$ and $I$ ), while the second items do not (i.e., $E$ and $L$; see Table 1). The structure in the non-adjacent two-way condition is identical to that used in the 'nonadjacent dependency' structure in the statistical learning literature, where results show evidence of learning (e.g., Gómez, 2005; Vuong, Meyer, \& Christiansen, 2016). Participants in the non-adjacent two-way condition only gain predictive information of the third item from the first item, while the second item will not help them. In the adjacent and non-adjacent two-way binding condition, both the first and second items independently predict the third item (see Table 1). The structure provides additional information to predict the third item compared to the traditional statistical learning task, where only the adjacent item (i.e., second item) provides predictive information of the third item (e.g., Saffran et al., 1996; Turk-Browne et al., 2012). Finally, the baseline condition 
consisted of all possible combinations of the items, and there was no way to predict the third items based on the two preceding items. The two two-way binding conditions resemble previous statistical learning studies where adults and even infants show evidence of learning (e.g. Gómez, 2002; Gómez \& Maye, 2005; Newport \& Aslin, 2004; Saffran et al., 1996; Turk-Browne et al., 2012; Vuong, Meyer, \& Christiansen, 2016), whereas we are not aware of any statistical learning research using a recombination of the same elements as in our three-way binding condition without confounds (cf., Cohen et al., 1990).

At test, participants were presented with congruent and incongruent triplets for the three experimental conditions. The congruent triplets were identical to the training triplets. The incongruent triplets used the same elements that were used in the training phase, but in new combinations so that the third item could not be predicted on the basis of learning the triplet structures in the training phase (see bottom section of Table 1). For example, for the congruent trials (or training trials) in the three-way condition, compound cues $X_{1} A$ or $X_{2} C$ predict item $B$, whereas these two compound cues in the incongruent trials would be followed by item $D$. For the baseline condition, there was no distinction between congruent and incongruent triplets since all possible combinations of elements were presented during training. Therefore, the baseline condition was only used to compare performance in the training phase to other conditions. Although the test phase of the baseline condition was not analyzed, in order to equate the length of each condition, filler triplets were used in the test phase of the baseline condition.

Each condition used a different set of pictures that were randomly assigned to each participant, and the stimuli were not reused across condition within subjects. Importantly, for 
all conditions, the frequency of each photo in each position was equated. For example, $X_{1}$ and $X_{2}$ were each repeated twice in the first position of the three-way condition, while $G, J, M, N$, repeated once in the second position of the adjacent and non-adjacent two-way condition (see Table 1). The frequency of males and females was also equated for each position. For example, in the three-way condition, if $X_{1}$ was randomly assigned to a female character, $X_{2}$ was assigned to a male character, and the same for $A$ and $C$, and $B$ and $D$. Five male and five female pictures which were not used in the main experiment were used for the practice phase.

\section{Procedure}

Participants were told that they would see images of cartoon characters one at a time, and their job was to distinguish whether each image was a male or a female character. They were also told to respond as quickly and accurately as possible. Images were presented on a white squared-patch subtending approximately $10^{\circ} \times 10^{\circ}$ of visual angle, which was centered on a black background of a 17-inch computer monitor. The image was presented until the participant made a response using the computer keyboard, and there was a $750 \mathrm{msec}$ interval filled with a black screen after each response.

There were four within-subjects conditions, where the order was randomized for each participant. The four conditions were identical except that the underlying presentation structure was different as explained in the previous section. Each condition consisted of a training phase and a test phase with a self-paced break between each condition. The procedure for the training phase and test phase was identical, and there were no breaks between the two phases making the transition unbeknownst to the participants (see Figure 3). The training phase consisted of 10 blocks for the three experimental conditions, and five blocks for the baseline condition. In 
each block, the training phase triplets were randomly presented once. At test, a mixture of congruent triplets (those presented in training) and incongruent triplets (i.e., new combinations of studied triplets) was presented. This made 144 trials for each condition (i.e., 120 training phase trials $(=3$ trials per triplet $\times 4$ triplet types per condition $\times 10$ repetitions; eight triplet types per condition $\times 5$ repetitions for the baseline condition $)+24$ test phase trials $(=3$ trials per triplet $\times 8$ triplet types per condition $[=4$ congruent +4 incongruent $]))$. A practice preceded the main experiment where the procedure was identical but only five male and five female characters that did not appear in the main experiment were presented in a random order.

\section{Results}

The analysis focused on the third item of each triplet since it is the critical position where the evidence of learning would be captured. Outlier participants were first identified by examining the total accuracy and correct reaction time (RT) during the learning phase. Three participants were excluded due to showing below-chance performance, and five participants were excluded based on extreme coefficient of variability $(\mathrm{CV})$ of their RTs $(\mathrm{CV}=\mathrm{SD} / \mathrm{Mean}$; Cheyne, Solman, Carriere, \& Smilek, 2009) which were outside of +2.5 SD of the group mean $\mathrm{CV}$. The total number of participants included in the analysis was 117 . In the following analyses, we report both frequentist and Bayesian analyses if possible. For the Bayesian analyses, unless reported otherwise, we used the JASP software (JASP Team, 2016) and calculated Bayes factors (BF) to evaluate null-effects (e.g., evidence for non-learning) especially for the test phase. We use $\mathrm{BF}_{10}$ to indicate Bayes factors favoring the alternative

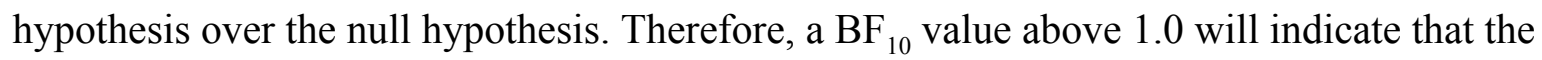
alternative is favored, whereas a value below one will indicate that the null hypothesis is 
favored. We also used the interpretation of Bayes factors by Jeffreys (1961), where a $\mathrm{BF}_{10}$ value between 1 and 3 is considered weak evidence, between 3 and 10 is considered substantial evidence, and above 10 is considered strong evidence for the alternative hypothesis. On the other hand, a $\mathrm{BF}_{10}$ value between 1 and $1 / 3$ is considered weak evidence, between $1 / 3$ and $1 / 10$ is considered substantial evidence, and below $1 / 10$ is considered strong evidence for the null hypothesis.

We first examined accuracy data. Participants showed accurate performance for all conditions during training $(M=.96, S D=.03)$, and accuracy was not different across the three experimental conditions nor throughout blocks (logistic regression with Conditions (3) and Block (10) as regressors did not reveal any effects or interactions, $p \mathrm{~s}>.17, B F_{10}=.08$ compared to the null model; here and elsewhere in the paper a method from Rouder, \& Morey (2012) was used to calculate all Bayes factors for the logistic regression model). Accuracy for the Baseline condition also did not change across the five blocks $\left(p=.74, B F_{10}=.17\right.$ compared to the null model). At test, accuracy was also high $(M=.96, S D=.05)$, with no evidence of difference between congruent and incongruent trials for all conditions (logistic regression with Congruence (2) as regressor did not reveal effects, $p \mathrm{~s}>.06, B F_{10} \mathrm{~s}<.195$ compared to the null model, see Figure 4).

Correct RTs in the training phase were first processed by calculating the median RT of each block for each participant in each condition (see top row of Figure 5). We first analyzed the difference in the first block across conditions in order to examine the amount of initial interference created. Results showed that RTs in the three-way condition $(M=603 \mathrm{~ms}, S D=$ $95 \mathrm{~ms})$ were significantly longer than in the baseline condition $(M=572 \mathrm{~ms}, S D=90 \mathrm{~ms}, p=$ 
$.003)$ and the non-adjacent two-way condition $(M=575 \mathrm{~ms}, S D=89 \mathrm{~ms}, p=.04)$. However, there was no evidence for a difference between the three-way condition and the adjacent and non-adjacent two-way $(M=585 \mathrm{~ms}, S D=90 \mathrm{~ms}, p=.34)$ condition (one-way repeated measures ANOVA using Greenhouse-Geisser correction, $F(2.89,334.84)=4.64, p=.004, \eta_{p}^{2}$ $=.038$, with Bonferroni post-hoc test; $B F_{10}=4.27$ compared to the null model). We also examined whether RTs in all conditions reached an asymptote during the training phase by examining the last three blocks of each condition. A Block (3) by Condition (4) repeated-measures ANOVA did not show any main effects nor interaction $(p s>.36$; the best non-null model was when only Condition was included in the model with $B F_{10}=.017$ compared to the null model), suggesting that RTs for all conditions reached an asymptote. The asymptote implies that no further learning should be expected with an increased number of training trials. Second, even though the asymptotes did not differ across the conditions, the three-way condition had a numerically higher asymptote $(M=549 \mathrm{~ms}, S D=73 \mathrm{~ms}$; calculated by the average of the last three blocks), compared to the other conditions (non-adjacent two-way, $M=537 \mathrm{~ms}, S D=80 \mathrm{~ms}$; adjacent and non-adjacent two-way, $M=543 \mathrm{~ms}, S D=94$ ms; baseline condition, $M=544 \mathrm{~ms}, S D=69 \mathrm{~ms}$ ). This result, coupled with the fact that the three-way condition had a longer RT in the first block, suggests that more interference may have been created in the $\mathrm{AB} / \mathrm{ABr}$ condition than other conditions.

To examine learning across the conditions, RTs in the test phase were processed (as in the training phase) by calculating the median RT of each block for each participant (see bottom row of Figure 5). A Condition (3) by Congruence (2) repeated-measures ANOVA showed a main effect of Congruence $\left(F=6.25, p=.014, \eta_{p}{ }^{2}=.051\right)$ and an interaction $(F=3.92, p=$ 
$\left..022, \eta_{p}^{2}=.033\right)$, but not for Condition $(F=.33, p=.71$; the best non-null model was when only Congruence was included in the model with $B F_{10}=1.05$ compared to the null model). To compare the effects across conditions, we further took the difference between the congruent and incongruent trials in each condition and conducted a one-way ANOVA. Results showed a main effect $\left(F=3.92, p=.02, \eta_{p}^{2}=.033 ; B F_{10}=1.70\right.$ compared to the null model $)$, where a post-hoc test with Bonferroni correction showed a statistical difference between the three-way condition and the adjacent and non-adjacent two-way condition $(p=.036$, difference $=33 \mathrm{~ms})$, a tendency for the non-adjacent two-way condition $(p=.099$, difference $=28 \mathrm{~ms})$, but not between the two two-way conditions ( $p=1.00$, difference $=4.02 \mathrm{~ms}$ ).

Since our main purpose of the current experiment was to compare the effect of congruence, we compared RTs for the congruent and incongruent trials in each condition. If the structure was learned after the training phase, the congruent trials will show faster RT than the incongruent trials. To evaluate evidence for non-learning (i.e., null-effect), we used Bayesian one-tailed paired t-tests using the JASP software (JASP Team, 2016), and calculated Bayes factors (BF) using a default Cauchy prior with a scale of .707. Results supported some evidence for learning in the non-adjacent two-way condition $\left(p=.012\right.$, Cohen's $d=.21 ; B F_{10}=$ 2.51) and strong evidence in the adjacent and non-adjacent two-way condition ( $p=.001$, Cohen's $\left.d=.28 ; B F_{10}=16.89\right)$. On the other hand, there was strong evidence of non-learning in the three-way condition $\left(p=0.79\right.$, Cohen's $\left.d=.08 ; B F_{10}=.06\right)$. We have examined the RT distributions at test in order to rule out the possible bimodality. However, we do not find any evidence of bimodality in our data across all experiments reported in the current study (see Appendix A for the actual distributions). 
In sum, the results show evidence for learning in the two two-way conditions and non-learning in the three-way condition. The former finding is consistent with previous statistical learning studies (e.g., Gómez, 2002; Turk-Browne et al., 2012), whereas the latter finding is a novel one. The evidence of non-learning, in conjunction with the fact that RTs during learning reached an asymptote in all conditions, suggest that three-way binding structures may not be learned implicitly and may require explicit attention. However, despite the learning curve asymptoting, it is possible that participants did not have sufficient trials to learn the three-way binding structures, and completing four within-subject conditions may have dropped the participants' motivation to learn the structures. Therefore, in Experiment 2, we examined whether additional learning trials (30 repetitions) will help the participants learn three-way binding structures with only testing the three-way condition.

\section{Experiment 2}

\section{Method}

Participants. One hundred undergraduates at The University of Melbourne participated for course credit. None of the subjects participated in Experiment 1. Eight additional participants were excluded due to experimenter error. The study was approved by The University of Melbourne Human Research Ethics Committee.

Materials, Design, and Procedure. Materials, design, and procedures were identical to Experiment 1 except that the participants experienced only the three-way condition, and there were 30 repetitions instead of 10 . This made 384 trials in total (i.e., 360 training phase trials $(=$ 3 trials per triplet $\times 4$ triplet types $\times 30$ repetitions $)+24$ test phase trials $(=3$ trials per triplet $\times 8$ triplet types $[=4$ congruent +4 incongruent $]))$. 


\section{Results}

We followed the same analyses as in Experiment 1. First, we excluded three participants that had $\mathrm{CV}$ greater than $+2.5 \mathrm{SD}$ of the group mean $\mathrm{CV}$, and one participant based on accuracy near chance. The total of 96 participants were included in the analysis. Overall accuracy during the learning phase was similar to Experiment $1(M=.96, S D=.03)$. Also, at test, there was no evidence of difference between congruent $(M=.91, S D=.14)$ and incongruent $(M=.94, S D=$ .11) trials (logistic regression with Congruency (2) as regressor did not reveal an effect, $p>$ $.07 ; B F_{10}=1.47$ compared to the null model).

Correct RTs in the training phase were first processed by calculating the median RT of each block for each participant in each condition (see Figure 6A). The learning curves reached an asymptote during the training phase (an one-way ANOVA with the last three blocks did not show a significant main effect, $F=1.87, p=.58 ; B F_{10}=.071$ compared to the null model). Importantly, at test, the difference between the congruent and incongruent trials showed strong evidence for non-learning (one-tail t-test; $p=.69$, Cohen's $d=.05 ; B F_{10}=.08$ ). As the learning curve was noisy, we analyzed the data with only including the participants who had CV scores lower than median CV value $(N=48$; see Figure $6 \mathrm{~B})$. However, the results showed evidence supporting non-learning $\left(p=.41\right.$, Cohen's $\left.d=.03 ; B F_{10}=.19\right)$. The result also did not change when only including the participants who had CV score lower than the lower quartile $\mathrm{CV}$ value $\left(N=25, p=.18\right.$, Cohen's $d=.19 ; B F_{10}=.52$; see Figure $\left.6 \mathrm{C}\right)$.

Experiment 2 replicates the three-way condition in Experiment 1 providing evidence that three-way bindings are not implicitly learnable. However, it is possible that the current task structure does not allow participants to learn the three-way structures even with explicit 
attention. Therefore, in Experiment 3, we manipulated the instructions where participants were explicitly told that there was a group of three and that they had to remember the three characters as a group.

\section{Experiment 3}

In Experiment 3, we examined whether three-way binding structures could be learned explicitly using the same paradigm used in Experiment 2. In addition to the original instructions, participants were told that three characters in a row formed a group and that they should try to remember the three as a group. We also alternated the background color between blue and red every three trials, and told the participants that whenever the color changes, a new group starts. The alternation of the background color was implemented to reduce the extra load in the current task compared to the implicit version of the task. In the current task, in addition to deciding whether the presented image was a male or female character, participants had to remember the three characters in a group, which required them to track the boundaries of the groups. We expected that the change in the background color would guide the participants to know where the boundary between the groups is.

\section{Method}

Participants. One hundred and one undergraduates at The University of Melbourne participated for course credit. None of the subjects participated in Experiment 1 or 2. One additional participant was excluded due to not understanding the instructions. The study was approved by The University of Melbourne Human Research Ethics Committee.

Materials, Design, and Procedure. Materials, design, and procedures were identical to Experiment 2 except that participants were additionally told that they should remember three 
characters that were presented consecutively within a group. We also alternated the background color between red and blue every three trials and told the participants that the change of background color indicates the start of a new group. The practice phase also contained nine trials instead of ten with the background changing as in the main training phase. The characters in the practice phase were not used in the main experiment.

\section{Results}

We first excluded one participant who had a CV greater than +2.5SD of the group mean $\mathrm{CV}$, resulting in 100 participants included in the analyses. Overall accuracy during the learning phase was similar to the previous experiments $(M=.96, S D=.04)$. At test, unlike the previous two experiments, there was evidence for difference between congruent $(M=.95, S D=.11)$ and incongruent $(M=.77, S D=.25)$ trials (logistic regression with Congruency (2) as regressor showed a statistically significant effect, $p<.001, B F_{10}=65.21$ compared to the null model). Correct RTs in the training phase were first processed by calculating the median RT of each block for each participant in each condition (see Figure 6D). The learning curves reached an asymptote during the training phase (an one-way ANOVA with the last three blocks did not show a significant main effect, $F=.32, p=.69 ; B F_{10}=.049$ compared to the null model). At test, the difference between the congruent and incongruent trials showed evidence for learning (one-tail t-test; $p<.001$, Cohen's $d=.37 ; B F_{10}=108$ ). In sum, Experiment 3 shows that with explicit instructions, the three-way structure is learned using the experimental paradigm used in the implicit version of the task. With both accuracy and RT showing robust effects for learning, the results are highly comparable to the non-learning results shown in Experiments 1 and 2. 


\section{General Discussion}

The current study examined whether complex three-way binding structures could be formed implicitly within a statistical learning task. The results showed evidence for non-learning in the three-way condition, which indicates that three-way binding structures may not be learned implicitly. The results were not due to insufficient training trials since training RTs in all conditions reached an asymptote (Experiment 1) and increasing the number of learning trials three fold did not allow participants to learn the three-way binding structure (Experiment 2). Moreover, the procedure did not preclude learning since there was evidence of learning in the other two-way conditions (Experiment 1) and with explicit instructions participants were able to learn three-way bindings using the same paradigm (Experiment 3). It is also worth noting that all three experiments were well powered with approximately 100 participants in each experiment.

Importantly, the findings provide a new constraint on the representational structure that can be learned through statistical/implicit learning. Previous studies show that participants can learn two-way bindings between two adjacent items or non-adjacent items in a statistical learning task (Santolin \& Saffran, 2018; Thiessen, Kronstein, \& Hufnagle, 2013), or associations among several items through multiple two-way bindings in a serial reaction time task (Nissen \& Bullemer, 1987). The current study shows that structures more complex than two-way bindings (e.g., three-way bindings) may not be learned implicitly. Constraints arising from the perceptual similarity of the items (e.g., Thiessen, 2011), or due to the lack of discriminability among items (e.g., Kirkham, Slemmer, Richardson, \& Johnson, 2007) have been reported previously in the statistical learning literature. However, providing evidence for 
a constraint on the complexity of the representation structure (i.e., three-way binding) is a novel finding. Considering that many theories of development and learning have been supported by statistical/implicit learning mechanisms (e.g., Gómez \& Gerken, 2000; Saffran \& Wilson, 2003; Vinter, Pacton, Witt, \& Perruchet, 2010), the implications of the current finding is not limited to statistical/implicit learning but extend to development and learning theories as well.

The results also provide an opportunity for models and theories to specify the chunking mechanisms more precisely. Computational models have been proposed to explain statistical/implicit learning as a general learning mechanism (e.g., French, Addyman, \& Mareschal, 2011; Mareschal \& French, 2017; Perruchet \& Vinter, 1998), and have used "chunking" as a general term throughout the literature. However, the results of the current study show that models of statistical learning should distinguish between different memory structures, and place constraints on what can and cannot be acquired during statistical learning.

Another interesting observation from the results shows how three-way interference may be ignored in an implicit learning task leading to non-learning. In the first block of the learning phase of Experiment 1, the three-way condition showed the highest RT implying that there is more interference than in other conditions. At the same time, the asymptotes of the conditions did not show a statistically significant difference, implying that the interference may have resolved to a degree. A possible explanation is that participants initially experienced interference from the preceding trials, but ignore them towards the end of the block, which resulted in no learning. If this is true, RT in the three-way condition at test (both congruent and incongruent) should be longer than the other learned conditions (i.e., congruent trials for 
the two two-way conditions), but faster than the incongruent trials. The bottom row of Figure 5 shows that this is numerically true. However, when running a one-way ANOVA over the six different conditions (i.e., three-way congruent, three-way incongruent, non-adjacent two-way congruent, non-adjacent two-way incongruent, adjacent and non-adjacent two-way congruent, and adjacent and non-adjacent two-way incongruent), we find a significant main effect $(F=$ $2.57, p=.026$; although null results from the Bayesian analysis, $B F_{10}=.30$ compared to the null model) only with a significant difference between the congruent and incongruent adjacent and non-adjacent two-way condition ( $p=.041$; Bonferroni post-hoc test). As the current study was mainly intended to examine whether there is learning or not at test, it is possible that the experimental design is not sensitive enough to capture the dynamics of the learning (or non-learning) process. A future study with a more sensitive paradigm would be required.

Finally, the result supports the hypothesis proposed by Yim et al. (2013) that explicit attentional control may be a critical prerequisite for forming three-way binding structures. Based on their results that the developmental change in the ability to form three-way bindings aligns with the protracted development of attentional control, Yim et al. (2013) hypothesized that explicit attentional control during encoding may be a key requirement in forming three-way bindings. As evidence of learning three-way structure was shown in Experiment 3 (under explicit attention instructions), the current study provides converging support to the hypothesis that explicit attention is critical in forming three-way binding structures. 


\section{References}

Armstrong, B. C., Frost, R., \& Christiansen, M. H. (2016). The long road of statistical learning research: past, present and future. Philosophical Transactions of the Royal Society B: Biological Sciences, 372(1711), 1-4.

Aue, W.R., Criss, A.H., \& Novak, M.D. (2017). Evaluating mechanisms of proactive facilitation in cued recall. Journal of Memory and Language, 94, 103-118.

Cheyne, J. A., Solman, G. J. F., Carriere, J. S. A., \& Smilek, D. (2009). Anatomy of an error: A bidirectional state model of task engagement/disengagement and attention-related errors. Cognition, 111(1), 98-113

Cohen, D. A., Ivry, R., \& Keele, S. W. (1990). Attention and structure in sequence learning. Journal of Experimental Psychology: Learning, Memory, and Cognition, 16(1), 17-30.

Doumas, L. A. A., Hummel, J. E., \& Sandhofer, C. M. (2008). A theory of the discovery and predication of relational concepts. Psychological Review, 115(1), 1-43.

Drummey, A. B., \& Newcombe, N. S. (2002). Developmental changes in source memory. Developmental Science, 5 (4), 502-513.

Elman, J. L. (1990). Finding structure in time. Cognitive Science, 14(2), 179-211.

Fiser, J., \& Aslin, R. N. (2002). Statistical learning of new visual feature combinations by infants. Proceedings of the National Academy of Sciences of the United States of America, 99(24), 15822-6.

Franco, A., Eberlen, J., Destrebecqz, A., Cleeremans, A., \& Bertels, J. (2015). Rapid Serial Auditory Presentation. Experimental Psychology, 62(5), 346-351.

French, R. M., Addyman, C., \& Mareschal, D. (2011). TRACX: a recognition-based 
connectionist framework for sequence segmentation and chunk extraction. Psychological Review, 118(4), 614-36.

Ghetti, S., \& Bunge, S. A. (2012). Neural changes underlying the development of episodic memory during middle childhood. Developmental Cognitive Neuroscience, 2 (4), 381-95.

Gómez, R. L. (1997). Transfer and complexity in artificial grammar learning. Cognitive Psychology, 33, 154-207.

Gómez, R. L. (2002). Variability and detection of invariant structure. Psychological Science, $13(5), 431-436$.

Gómez, R. L., \& Gerken, L. (2000). Infant artificial language learning and language acquisition. Trends in Cognitive Sciences, 4(5), 178-186.

Gómez, R. L. \& Maye, J. (2005). The Developmental Trajectory of Nonadjacent Dependency Learning. Infancy, 7, 183-206.

Halford, G. S., Wilson, W. H., \& Phillips, S. (1998). Processing capacity defined by relational complexity: Implications for comparative, developmental, and cognitive psychology. Behavioral and Brain Sciences, 21, 803-865.

Humphreys, M. S., Bain, J. D., \& Pike, R. (1989). Different ways to cue a coherent memory system: A theory for episodic, semantic, and procedural Tasks. Psychological Review, 96 (2), 208-233.

JASP Team. (2016). JASP (Version 0.8.0.0) [Computer software]. Retrieved from 
https://jasp-stats.org/.

Jeffreys, H. (1961). Theory of probability (3rd Ed.). Oxford, UK: Oxford University Press.

Kirkham, N. Z., Slemmer, J. A., \& Johnson, S. P. (2002). Visual statistical learning in infancy: evidence for a domain general learning mechanism. Cognition, 83, B35-B42.

Kirkham, N. Z., Slemmer, J. A., Richardson, D. C., \& Johnson, S. P. (2007). Location, location, location: Development of spatiotemporal sequence learning in infancy. Child Development, 78(5), 1559-1571.

Leys, C., Ley, C., Klein, O., Bernard, P., \& Licata, L. (2013). Detecting outliers: Do not use standard deviation around the mean, use absolute deviation around the median. Journal of Experimental Social Psychology, 49(4), 764-766.

Mareschal, D., \& French, R. M. (2017). TRACX2: a connectionist autoencoder using graded chunks to model infant visual statistical learning. Philosophical Transactions of the Royal Society B: Biological Sciences, 372(1711), 20160057.

Minsky, M. L., \& Papert, S. A. (1969). Perceptrons. Cambridge, MA: MIT Press

Newport, E. L., \& Aslin, R. N. (2004). Learning at a distance I. Statistical learning of non-adjacent dependencies. Cognitive Psychology, 48(2), 127-162.

Nissen, M. J., \& Bullemer, P. (1987). Attentional requirements of learning: Evidence from performance measures. Cognitive Psychology, 19 (1), 1-32.

Osth, A. F., \& Dennis, S. J. (2015). Sources of interference in item and associative recognition memory. Psychological Review, 122(2), 260-311.

Porter, L. W., \& Duncan, C. P. (1953). Negative Transfer in Verbal Learning. Journal of Experimental Psychology, 46 (1), 61-64. 
Perruchet, P., \& Vinter, A. (1998). PARSER: a model for word segmentation. Journal of Memory and Language, 39, 246-263.

Plate, T. A. (1995). Holographic reduced representations. IEEE Transactions on Neural Networks, 6(3), 623-641.

Reber, A. S. (1967). Implicit learning of artificial grammars. Journal of Verbal Learning and Verbal Behavior, 6 (6), 855-863.

Rouder, J. N., \& Morey R. D. (2012). Default Bayes factors for Model Selection In Regression. Multivariate Behavioral Research. 47, 877-903

Saffran, J. R., Aslin, R. N., \& Newport, E. L. (1996). Statistical learning by 8-month-old infants. Science, 274, 1926-1928.

Saffran, J. R., \& Kirkham, N. Z. (2018). Infant Statistical Learning. Annual Review of Psychology, 69(1), 181-203.

Saffran, J. R., Newport, E. L., Aslin, R. N., Tunick, R. A., \& Barrueco, S. (1997). Incidental Language Learning: Listening (and Learning) Out of the Corner of Your Ear. Psychological Science, 8(2), 101-105.

Saffran, J. R., \& Wilson, D. P. (2003). From syllables to syntax: Multilevel statistical learning by 12-month-old infants. Infancy, 4(2), 273-284.

Santolin, C., \& Saffran, J. R. (2018). Constraints on Statistical Learning Across Species. Trends in Cognitive Sciences, 22(1), 52-63. 
Siegelman, N., Bogaerts, L., Christiansen, M. H., \& Frost, R. (2017). Towards a theory of individual differences in statistical learning. Philosophical Transactions of the Royal Society of London: Series B, Biological Sciences, 372(1711), 20160059.

Sloman, S., \& Rumelhart, D. (1992). Reducing Interference in Distributed Memory Through Episodic Gating. In A. F. Healy, S. M. Kosslyn, \& R. M. Shiffrin (Eds.) From Learning Theory to Connectionist Theory: Essays in Honor of William K. Estes, (pp. 227-248). Hillsdale, NJ: Lawrence Erlbaum Associates, Inc

Thiessen, E. D. (2011). Domain General Constraints on Statistical Learning. Child Development, 82(2), 462-470.

Thiessen, E. D., Kronstein, A. T., \& Hufnagle, D. G. (2013). The extraction and integration framework: A two-process account of statistical learning. Psychological Bulletin, 139(4), $792-814$.

Turk-Browne, N. B., Simon, M. G., \& Sederberg, P. B. (2012). Scene representations in parahippocampal cortex depend on temporal context. The Journal of Neuroscience, 32 (21), 7202-7207.

Vinter, A., Pacton, S., Witt, A., \& Perruchet, P. (2010). Implicit learning, development, and education. Rethinking physical and rehabilitation medicine: New technologies induce new learning strategies. In J.-P. Didier \& E. Bigand (Eds.) (pp. 111-127). Paris: Springer Paris.

Vuong, L. C., Meyer, A. S., \& Christiansen, M. H. (2016). Concurrent Statistical Learning of Adjacent and Nonadjacent Dependencies. Language Learning, 66(1), 8-30. 
Wahlheim, C. N., \& Jacoby, L. L. (2013). Remembering change: The critical role of recursive remindings in proactive effects of memory. Memory \& Cognition, 41(1), 1-15.

Wiles, J., \& Humphreys, M. S. (1993). Using Artificial Neural Nets to Model Implicit and Explicit Memory Test Performance. In P. Graf, \& M. E. Masson (Eds.) Implicit Memory: New Directions in Cognition, Development, and Neuropsychology, (pp. 141-166). New York, New York, USA: Lawrence Erlbaum Associates, Inc.

Yim, H. (2019, November 20). Examining Three-way Binding as a Constraint on Statistical Learning. Retrieved from osf.io/48ymf

Yim, H., Dennis, S. J., \& Sloutsky, V. M. (2013). The Development of Episodic Memory: Items, Contexts, and Relations. Psychological Science, 24 (11), 2163-2172.

Yim, H., Osth, A. F., Sloutsky, V. M., \& Dennis, S. J. (2018). Evidence for the use of three-way binding structures in associative and source recognition. Journal of Memory and Language, 100, 89-97. 
Table 1

Triplet structure for each condition used in the experiments. All four conditions were used in Experiment 1, whereas only the Three-way condition was used in Experiments 2 and 3. Each element represents images of a unique cartoon character.

\begin{tabular}{|c|c|c|c|c|c|}
\hline & Three-way & $\begin{array}{l}\text { Non-adjacent } \\
\text { two-way }\end{array}$ & $\begin{array}{c}\text { Adjacent and } \\
\text { non-adjacent } \\
\text { two-way }\end{array}$ & & Baseline \\
\hline \multirow[t]{8}{*}{ Learning Phase } & $X_{1-A}-B$ & $X_{3}-E-F$ & $X_{5-G-H}$ & Learning Phase & $X_{7-O}-P$ \\
\hline & $X_{1-C-D}$ & $X_{3-L-F}$ & $X_{5-M-H}$ & & $X_{7-Q-P}$ \\
\hline & $X_{2}-A-D$ & $X_{4-E-I}$ & $X_{6-J-K}$ & & $X_{7-O}-R$ \\
\hline & $X_{2}-C-B$ & $X_{4-L-I}$ & $X_{6-N}-K$ & & $X_{7-Q}-R$ \\
\hline & & & & & $X_{8-O}-P$ \\
\hline & & & & & $X_{8-Q}-P$ \\
\hline & & & & & $X_{8-O}-R$ \\
\hline & & & & & $X_{8-Q} Q-R$ \\
\hline \multirow{4}{*}{$\begin{array}{l}\text { Test Phase } \\
\text { (incongruent trials) }\end{array}$} & $X_{1-A}-D$ & $X_{3}-E-I$ & $X_{5-G-K}$ & Test Phase & $O-X_{7-} P$ \\
\hline & $X_{1-C}-B$ & $X_{3-L-I}$ & $X_{5-M-K}$ & (filler trials) & $Q-X_{7-R}$ \\
\hline & $X_{2}-A-B$ & $X_{4-E-F}$ & $X_{6-J}-H$ & & $O-X_{8}-Q$ \\
\hline & $X_{2-C}-D$ & $X_{4-L-F}$ & $X_{6-N}-H$ & & $Q-X_{8}-P$ \\
\hline
\end{tabular}


A Learning sequence $\quad 1-3-2-3-1-2-1-3-2-3-1-2 \cdots$

B 1-back predictions 2-back predictions

$$
\begin{aligned}
& 1 \rightarrow 3 \\
& 1 \rightarrow 2 \\
& 2 \rightarrow 3 \\
& 2 \rightarrow 1 \\
& 3 \rightarrow 1 \\
& 3 \rightarrow 2
\end{aligned}
$$

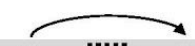$$
1 \longdiv { 1 }
$$

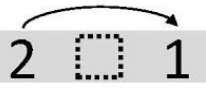$$
2 \longdiv { 1 3 }
$$$$
3 \longdiv { 2 }
$$

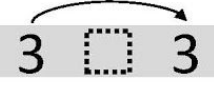

C

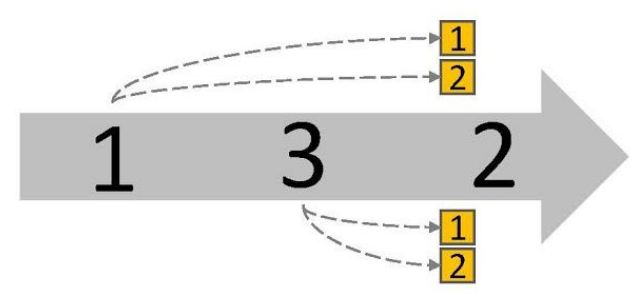

D

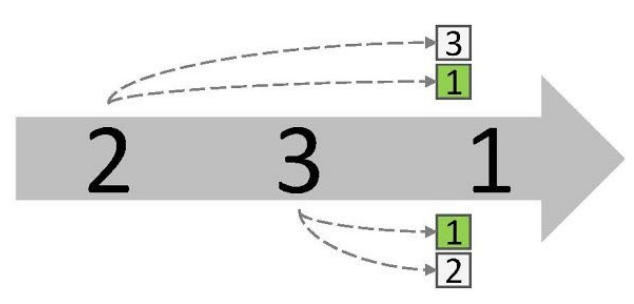

Figure 1. An illustration of the sequence structures used in Cohen, Ivry, and Keele (1990). (A) an example of the learning sequence, (B) all possible 1-back and 2-back predictions that can be learned through the sequence, $(C)$ a sequence that requires a three-way binding, and (D) a sequence that does not require a three-way binding. 


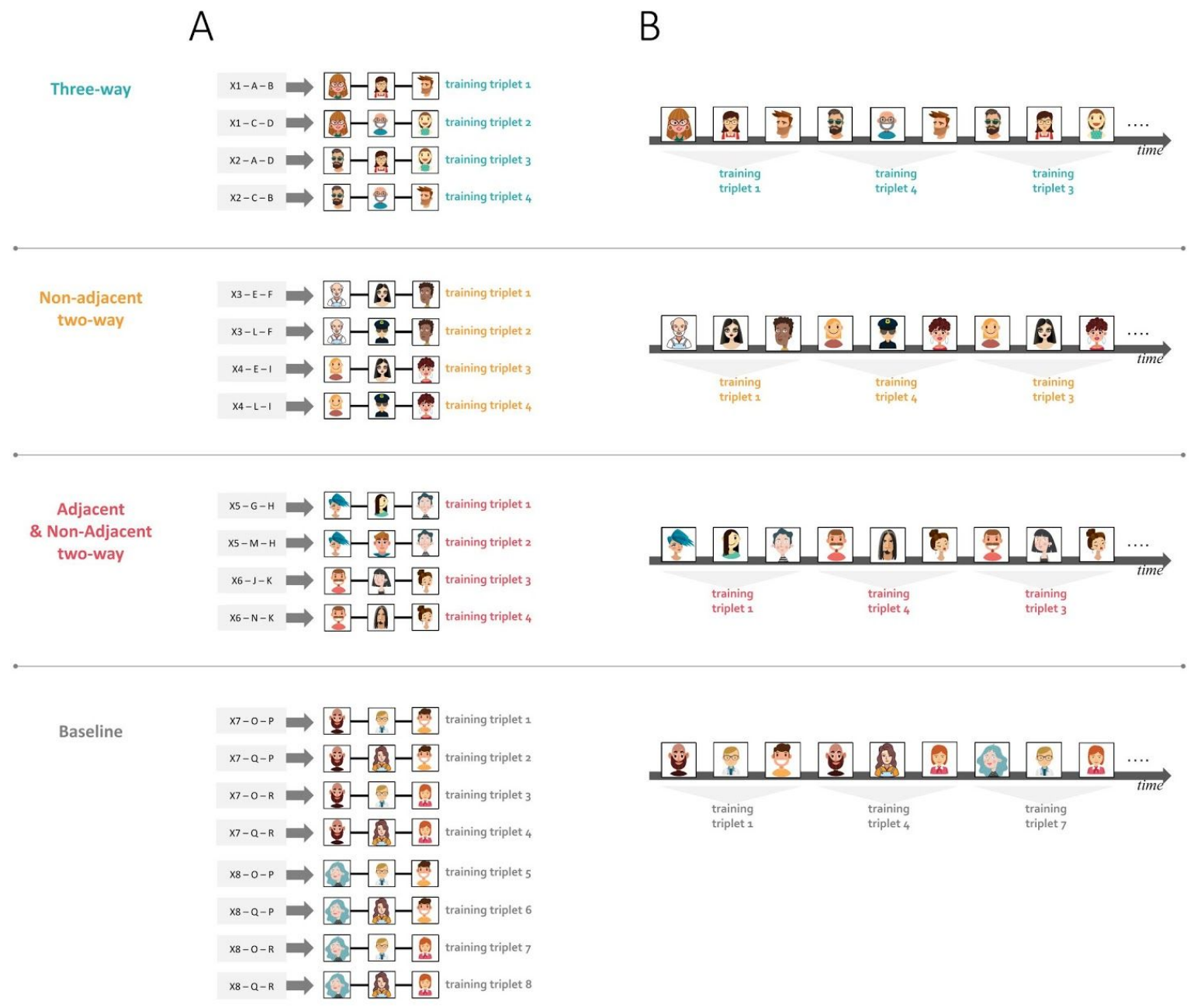

Figure 2. Different structures used for different conditions in the experiments and illustrating the modification of the structures into a statistical learning task -- all four conditions were used in Experiment 1, whereas only the three-way condition was used in Experiment 2 and 3. Each row shows different structures from Table 1 and how it is modified into a statistical learning task in the current experiment. Column (A) shows how each event in the paired-associate learning task is taken as a triplet and are represented as cartoon characters, and column (B) shows how each photo was presented one at a time to the participants, where participants were told to decide whether the character was a male or female. Note that the above figure uses example cartoon characters whereas popular cartoon characters (e.g., Dora the Explorer, Bart Simpson), were used in the actual experiments (image: Freepik.com). 


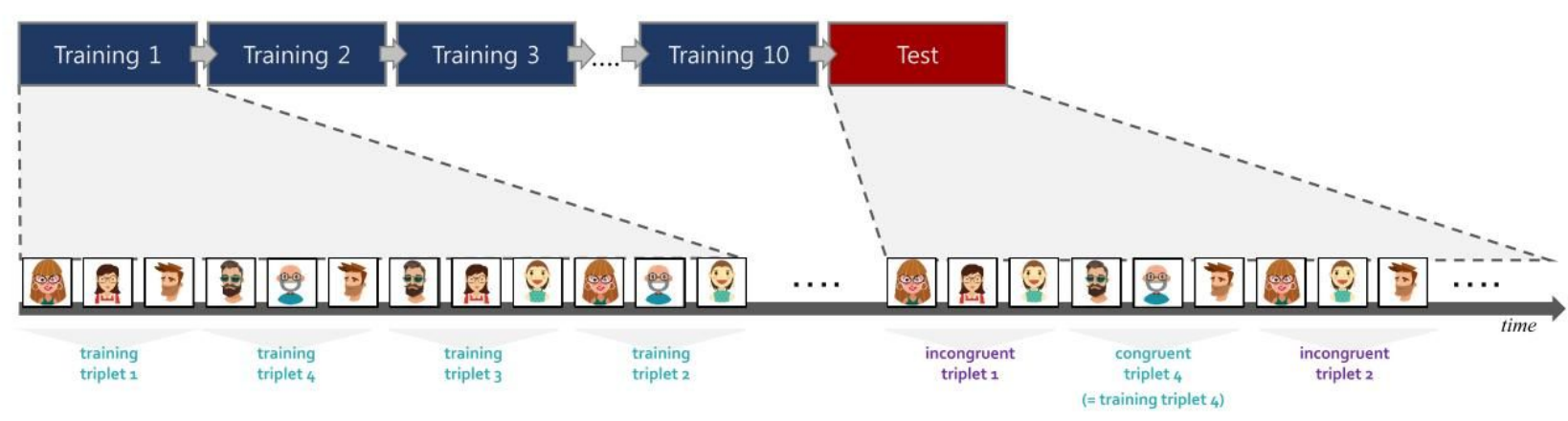

Figure 3. An example of the three-way condition of the task in Experiment 1. There were ten training blocks and one test block. There were no breaks between the blocks in that the participants were not aware of the transitions. In each training block, the training triplets repeated once in a random order, while in the test block, training triplets (i.e., congruent triplets) and incongruent triplets were randomly repeated once. The participant's job was to decide whether the photo was a male or female. In Experiment 2 and 3, the length of the training blocks increased from 10 to 30 . Note that the above figure uses example cartoon characters whereas popular cartoon characters (e.g., Dora the Explorer, Bart Simpson), were used in the actual experiments (image: Freepik.com). 

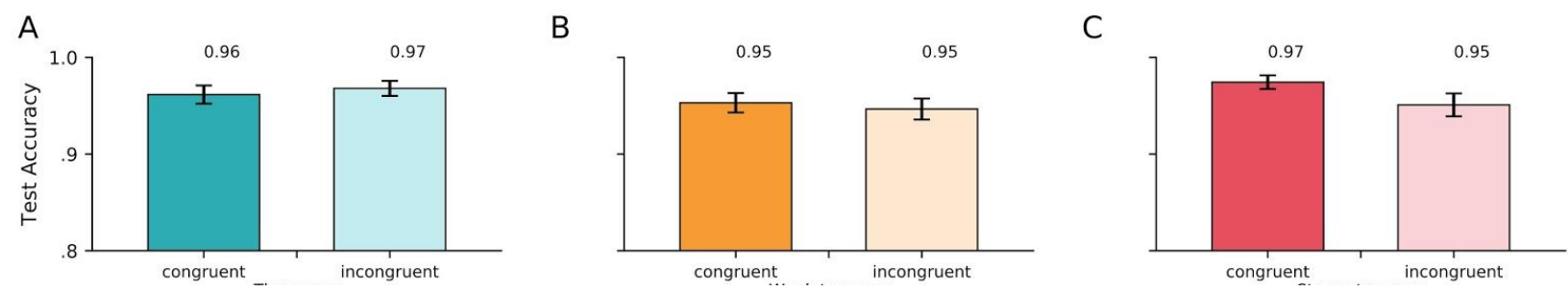

Figure 4. Accuracy results from the test phase in Experiment 1. (A) Three-way binding, (B) Non-adjacent two-way binding, and (C) Adjacent and non-adjacent two-way binding. Error bars represent \pm 1 SEM. 
A $\quad$ B
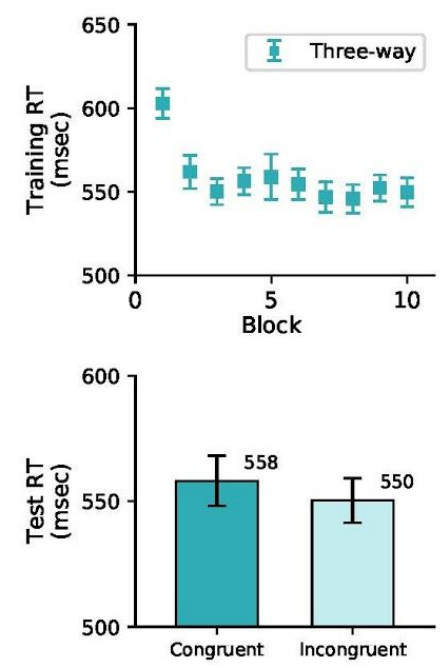
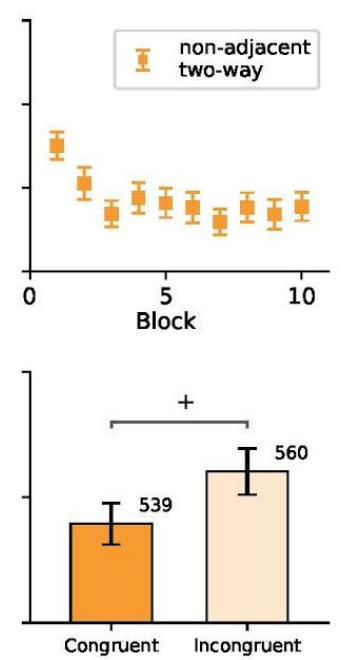

C
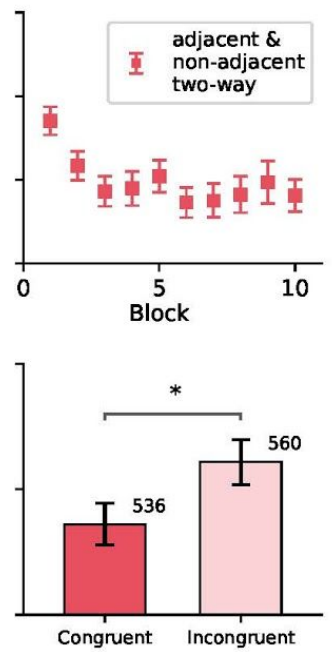

D

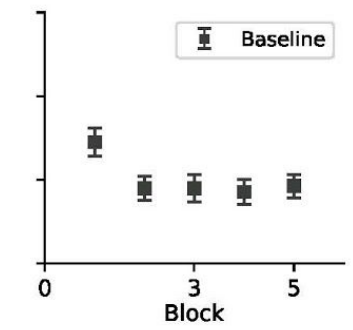

Figure 5. Reaction time (RT) results from Experiment 1. The top row shows RTs from the training phase, and the bottom row shows RTs from the congruent and incongruent triplets in the test phase. Each column shows a different condition - (A) three-way binding, (B) non-adjacent two-way binding, (C) adjacent and non-adjacent two-way binding, and (D) Baseline. Error bars represent \pm 1 SEM. + and $*$ indicate $p=.012$ and $p=.001$ respectively. 


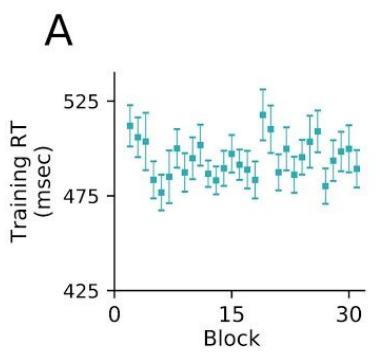

B
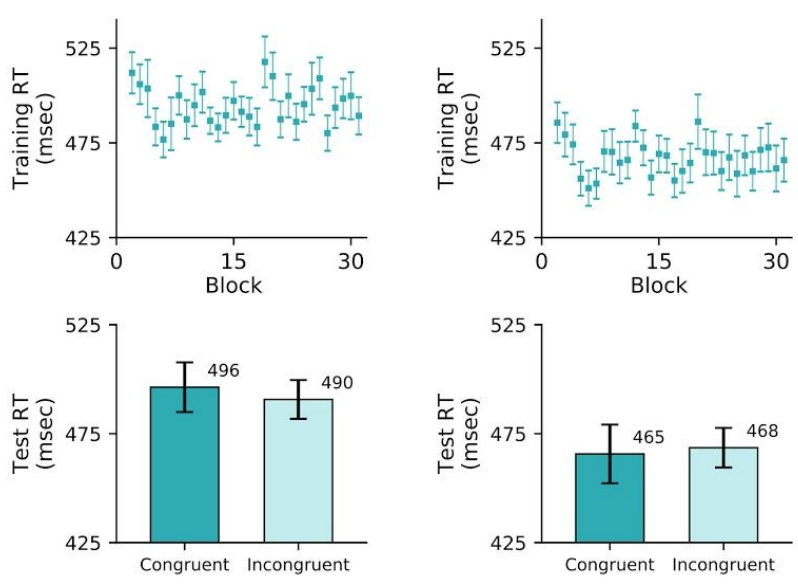

C
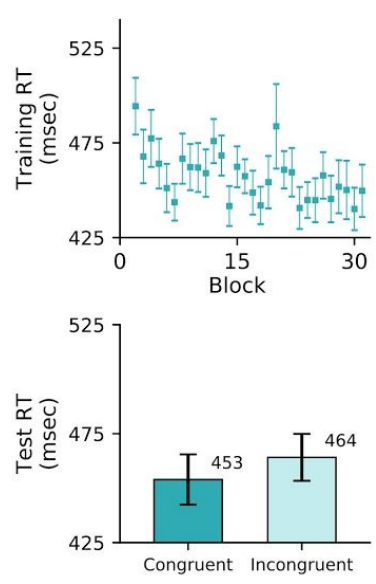
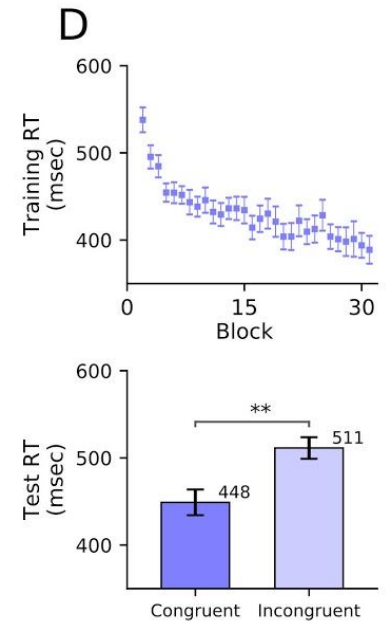

Figure 6. Results from Experiment 2 (three-way implicit condition) and 3 (three-way explicit condition), where the top row shows RT during learning and the bottom row shows RT at test. (A) Experiment 2 with all participants included, (B) Experiment 2 with participants who have $\mathrm{CV}$ less than the median CV value included, (C) Experiment 2 with participants who have CV less than the lower quartile of the CV included. (D) Experiment 3. Error bars represent \pm 1 SEM, and ** indicates $p<.001$. 
Appendix A.
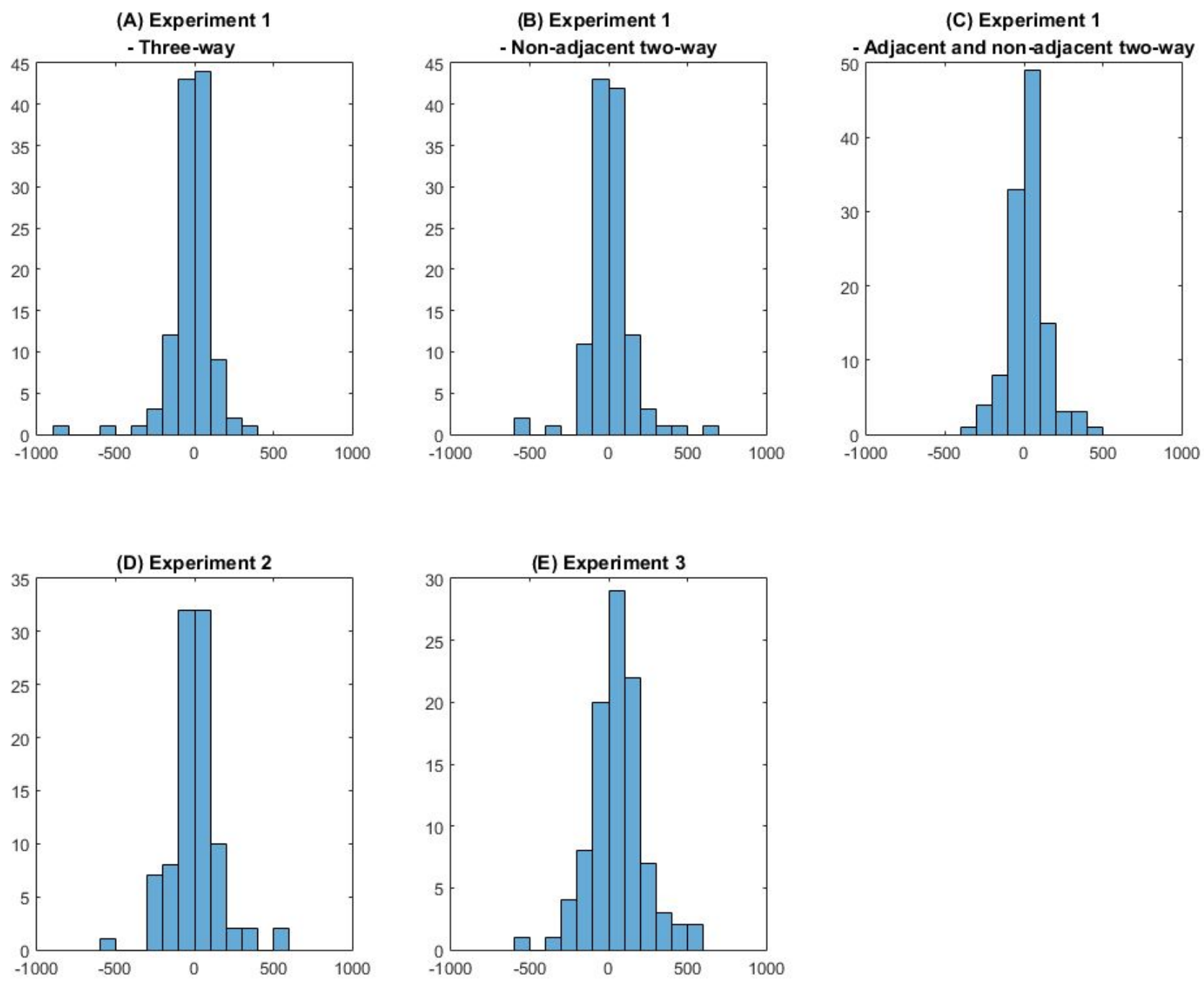

Figure A1. Reaction time distributions (individual incongruent condition minus individual congruent condition) at test. (A) Three-way binding condition in Experiment 1, (B) Non-adjacent two-way binding condition in Experiment 1, (C) Adjacent and non-adjacent two-way binding condition in Experiment 1, (D) Experiment 2, and (E) Experiment 3. 